UCRL-ID-120523

\title{
Preliminary 2D Design Study for A\&PCT
}

\section{Eric Keto, Steve Azevedo, Pat Roberson, Dan Deckman,} Harry Martz, K. Brown, and B. Hasegawa

\section{RECEIVED \\ OCT 061995 \\ OSTI}

March 1995

This is an informal report intended primarily for internal or limited external distribution. The opinions and conclusions stated are those of the author and may or may not be those of the Laboratory. 


\section{DISCLAIMER}

This document was prepared as an account of work sponsored by an agency of the United States Government. Neither the United States Government nor the University of California nor any of their employees, makes any warranty, express or implied, or assumes any legal liability or responsibility for the accuracy, completeness, or usefulness of any information, apparatus, product, or process disclosed, or represents that its use would not infringe privately owned rights. Reference herein to any specific commercial product, process, or service by trade name, trademark, manufacturer, or otherwise, does not necessarily constitute or imply its endorsement, recommendation, or favoring by the United States Government or the University of California. The views and opinions of authors expressed herein do not necessarily state or reflect those of the United States Government or the University of California, and shall not be used for advertising or product endorsement purposes.

This report has been reproduced directly from the best available copy.

Available to DOE and DOE contractors from the Office of Scientific and Technical Information P.O. Box 62, Oak Ridge, TN 37831

Prices available from (615) 576-8401, FTS 626-8401

Available to the public from the National Technical Information Service

U.S. Department of Commerce 5285 Port Royal Rd. Springfield, VA 22161 


\section{DISCLAIMER}

\section{Portions of this document may be illegible in electronic image products. Images are produced from the best available original document.}




\title{
Preliminary 2D Design Study for A\&PCT
}

Eric Keto, Steve Azevedo, Pat Roberson, Dan Decman, and Harry Martz Lawrence Livermore National Laboratory

Livermore, California

and

Keenan Brown and Bruce Hasegawa

University of California at San Francisco

San Francisco, California

\begin{abstract}
Lawrence Livermore National Laboratory is currently designing and constructing a tomographic scanner to obtain the most accurate possible assays of radioactivity in barrels of nuclear waste in a limited amount of time. This study demonstrates a method to explore different designs using laboratory experiments and numerical simulations. In particular, we examine the trade-off between spatial resolution and signal-to-noise. The simulations are conducted in two dimensions as a preliminary study for three dimensional imaging. We find that the optimal design is entirely dependent on the expected source sizes and acitvities. For nuclear waste barrels, preliminary results indicate that collimators with widths of 1 to 3 " and aspect ratios of 5:1 to 10:1 should perform well. This type of study will be repeated in $3 \mathrm{D}$ in more detail to optimize the final design.
\end{abstract}

\section{Introduction}

This study is part of a larger design effort to construct a tomographic scanner for the accurate assay of radioactivity in barrels of nuclear waste. The scanner is a follow on to an earlier version which demonstrated the capabilities of tomography for nuclear waste assays [ROB94]. This study, focuses on those design aspects which relate to imaging and assay accuracy. These include both hardware and software issues, specifically, the width and length of the collimators, the sampling pattern, and the type of image reconstruction algorithm. This report will describe some preliminary choices based on trials of different designs.

*Work performed under auspices of the U.S. Department of Energy by the Lawrence Livermore National Laboratory under contract No. W-7405-ENG-48. 
The scanner under development will use both active and passive computed tomography (A\&PCT), sometimes called transmission and emission tomography. A\&PCT is a two step process [MAR92]. In the first step we tomographically construct an image of the mass in the barrel in terms of the mass absorption to penetrating radiation. This is done by measuring the attenuation of a calibrated radioactive $166 \mathrm{mHo} 3 \mathrm{mCi}$ source (active) along a large number of lines of sight through the waste barrel. This is identical in principle to CAT imaging commonly done in medicine. In the second step we measure the radiation emitted by the radioactive waste itself (passive) and tomographically construct an image of the radioactive sources in the barrel taking account of the absorption of outgoing radiation by mass absorbers in the barrel. This is similar in principle to medical SPECT imaging. In our project we are really only interested in the total activity of the barrel and not in the images of the contents. Nonetheless for an accurate assay it is necessary to form images of both the mass absorbers and emitters so that the measured activity may be properly corrected for absorption.

Accurate imaging is thus a requirement for accurate assays. The accuracy of imaging or image quality may be variously assessed. In this study we will be concerned primarily with spatial resolution and signal-to-noise, $\mathrm{S} / \mathrm{N}$, since these qualities, which directly affect the accuracy of the final assay, are themselves directly affected by the collimator design. In general there is a steep trade off between spatial resolution and $S / N$ in the hardware because designs which offer high spatial resolution, that is collimators with high aspect ratio, will always collect relatively fewer photons per detector. The $\mathrm{S} / \mathrm{N}$ in turn scales as the root of the number of photons. A significant conclusion of this report is a demonstration of one method to explore the trade-off between $S / N$ and spatial resolution.

This is a preliminary design study. Specifically the study is done with so called two dimensional imaging. This means that the data acquisation and the image reconstruction deal with only one plane or slice through the waste barrrel at a time. While reconstructing a single slice, the algorithm assumes that the adjacent slices have exactly the same structure as the slice under reconstruction. We can construct laboratory experiments satisfying this assumption, for example, measuring the activity of a long radioactive rod placed vertically in a barrel. Working in only two dimensions allows both the hardware experiments and the numerical reconstruction to be accomplished much faster than in full three dimensional imaging. For example in assaying a long rod, it is necessary to measure the emission in only one slice near the center of the rod, all other slices being presumed equal. 
However, in a barrel of real waste, the assumption of equal adjacent slice planes will not often be correct. This will occur in our rod example at the ends of the rod. Although three dimensional images of sources which vary in the vertical direction can be built up out of a stack of separate $2 \mathrm{D}$ images, full three dimensional imaging which measures and correctly accounts for the differences between slices will probably be required for the most accurate assays. Nonetheless, for expediency, we chose to begin our design study with two dimensional imaging. We expect that the evaluation techniques which we develop to test hardware and software in 2D will be applicable to three dimensional imaging and assaying although the results might differ somewhat.

This report describes the results of three types of trials, laboratory experiments, involving real sources and hardware, and two levels of numerical simulation. The laboratory experiments use much of the hardware and electronics which will be in the final version of the A\&PCT system. The source is a long ${ }^{133} \mathrm{Ba} 1 \mathrm{mCi}$ rod chosen to match the $2 \mathrm{D}$ assumptions of the trial. The laboratory experiments gives us a first assessment of the potential accuracy of a tomographic scanner including all sources of error in the system, but are the most difficult of the three trials. Thus we use only the simplest geometry in the laboratory experiments. The first level of numerical simulation uses a sophisticated Monte Carlo transport code to generate simulated data. The primary purpose of the Monte Carlo modeling is to compare data and reconstructions simulated by a sophisticated code with data and reconstructions simulated by a much simpler multi-ray tracing model. It is this second simpler level of modeling that we will use to explore different hardware designs. Again this is a question of expediency. The multi-ray models are simple to construct and fast to execute. Full Monte Carlo simulations of a large number of trial hardware designs would be prohibitively time consuming. In any case, our limited Monte Carlo modeling confirms the validity of our simpler multi-ray tracing models as described below.

\section{Reconstruction Code}

The two step A\&PCT imaging requires two image reconstruction codes, one to reconstruct the image of the absorbers from the active measurements, and the second to reconstruct the image of the emitters from the passive measurements. For the active tomography we use a reconstruction code developed at Livermore [AZE90] based on the Filtered Back Projection (FBP) algorithm. In reconstructing the image of the emitters, the reconstruction must model both emission and absorption, so the FBP algorithm is no longer appropriate. We use an emission reconstruction code 
developed at the University of California, San Francisco [BRO94]. This reconstruction code uses a Maximum Likelihood-Expectation Maximization (MLEM) algorithm [SHE82] and models emission, absorption, and the collimator response by following photons along a number of rays (multi-ray) through the source to the detector. We use a two dimensional version of this code in all the experiments in this study, both laboratory and experimental.

\section{Experiments}

A simple laboratory experiment was designed to validate the simulations and provide an approximate accuracy for the A\&PCT system. The source was a thin barium rod, 6" long, with a total activity of 890 microCuries. This was placed $5^{\prime \prime}$ from the center of rotation, and scanned with a detector looking through a 1" square collimator 20" long, 30" away from the center of rotation.. The detector itself is a P-type coaxial high purity germanium (HPGe) detector 3" in diameter and 3" deep [ROB94]. The field of view at the center of rotation is about $3.5^{\prime \prime}$ square. A 4 " diameter aluminum pipe with a $1 / 2$ " thick wall was placed around the rod to introduce some absorption into the experiment. We imaged the $356 \mathrm{keV}$ line of barium with an integration time of 30 seconds per detector position. Our assay of the rod was accurate to within $7 \%$.

Our Monte Carlo simulations used the LANL code MCNP [BRI86]. For the Monte Carlo simulations, the source was placed at the center of rotation so that only one view needed to be simulated. A sinogram was created by replicating this simulation over a number of angles. Four experiments were run, low and high resolution, with and without absorption. The low resolution experiments simulated a 2 " square collimator $10^{\prime \prime}$ long, and the high resolution a $1 / 4$ " square collimator 5 "long. The experiments were run with and without a simulated 4 " diameter aluminum tube with a 1 " thick wall around the line source. Using the UCSF MLEM reconstruction code, our assays were accurate to within $4 \%$ for all cases. All the Monte Carlo simulations were run with enough photons so that the statistical noise is negligible.

We compared the Monte Carlo data with data produced by the multi-ray simulator. For the multi-ray simulator, images of the sources and absorbers were produced on a grid 8 times finer than the collimator width with a sufficient number of rays to pass through every pixel of the grid. Sinograms produced by both simulators were accurate to within a few percent in integrated counts, the only difference being in the exact shape of the instrument response function. At high resolution, some differences are 
apparent. At lower resolution, the multi-ray simulator assumes a far field approximation to the response function which is triangular rather than trapezoidal (figure 1). The newer 3D code which we will use with our A\&PCT system will use the exact response function. In any case, the multiray simulator is capable of generating quantitatively accurate sinogram data for our simple experiments.

\section{Hardware Trade off between spatial resolution and signal-to-noise.}

We used the multi-ray simulator for our hardware design study. The central issue in the hardware design concerns the appropriate spatial resolution of the system and the trade off between resolution and the signal-to-noise ratio of the images. Our simulated trials of preliminary hardware design study focus on demonstrating one method to investigate this trade off. This trade off arises because higher resolution systems will always receive fewer photons than low resolution designs. Since the emission of radiation is characterized by Poisson statistics, the signal-to-noise ratio scales as the square root of the number of photons detected in the measurement. For a point source or a number of well separated point sources, the number of photons detected given a fixed period of time to scan an entire barrel scales as the 5th power of the resolution. For example, if we increase the resolution by reducing the collimator width from 2 " to 1 " in both width and height, the active area of the detector and the number of photons detected will decrease by the square of the improvement in resolution. If we now consider that with a single 1 " square collimator rather than a $2 "$ ", we will need four times as many separate measurements to completely cover one view of the waste barrel we will have less time to spend on each measurement. This accounts for another factor of the resolution squared. Finally, we need to observe the drum from more different angles to fully realize the improvement in resolution. In this study the number of angular views is 1.5 times the number of detector positions required to cross the object. The number of views accounts for the final fifth factor. Thus in all, the photon counts from a point source will scale with the 5th power of the resolution and the signal to noise will scale as the root of 5 , the 2.5 th power. By the same considerations, the number of photon counts from a large uniform source scales as the 7th power of the resolution. The extra power of two comes from the fact that as the resolution is lowered, the field of view of the detector increases encompassing a greater volume of the radioactive source resulting in more photon counts. 
For a given amount of time to scan an entire barrel, we can expect that at higher resolution the errors in the assay will be dominated by statistical errors related to low signal to noise. At lower resolution, the errors in the assay will be dominated by a blurring of the emission and absorption regions, so called partial volume errors. At some resolution in between we should be able to obtain the most accurate assay. This relationship is depicted in figure 2. The curved lines represent the maximum probable error due either to resolution or statistics. This optimum resolution, least maximum probable error, will depend on the one hand on the spatial resolution relative to the expected sizes of the radioactive sources and the absorbers, and on the other on the expected number of counts.

While the waste barrels may in general contain just about anything, any size and density absorbing object and any activity, we can nonetheless make some estimates useful toward deciding on an optimum collimator width and length. For example, the size of the source can certainly be no bigger than the barrel itself, about 24 " in diameter. We might expect that at a minimum, the aperture ought to be small enough, 2 " to 4 ", to resolve the shape of the barrel itself. For an expected activity we may use the definition of the maximum threshold for low level waste, $100 \mathrm{nCi} / \mathrm{gm}$. For example, the expected number of counts from a uniform source of $100 \mathrm{nCi}$ of $\mathrm{Pu}$ in each gram of a 24" diameter glass cylinder assuming we are measuring the 414$\mathrm{keV}$ line with a $2 "$ square detector and a $10^{\prime \prime}$ collimator is 1 count per second. Thus fairly simple considerations suggest that the collimator width should be 1 to 4 ". A width much smaller would not receive enough counts to generate a meaningful image, while one much larger would not even resolve the barrel itself.

The collimator width is one question we will address in our preliminary hardware design study. In addition we will be interested in the collimator length and the sampling interval. The sampling interval refers to the spacing between measurements. If the detector spacing is close, then as with most optical instruments, the field of view widens as we get further from the detector, we may be viewing the same parts of the drum with several detector positions. If the spacing is too coarse, we may miss some of the drum and be unable to construct a full image. Small objects could escape detection. However, with coarser spacing, we will have more time to spend on each measurement which will improve the $S / N$ of those measurements The question is again one of trading off spatial resolution for $S / N$. 
We can make all the questions more precise with some numerical simulations of the accuracy of different hardware designs. In a numerical simulation we can test a specific hardware design with a source of specific activity and shape. Different hardware designs will perform better with some types of sources than with others. To get an understanding of overall performance we need to perform our experiments on a variety of sources. The simulated sources were designed on two criteria. First, the sources should be broadly representative of the types of radioactive sources which might be found in typical waste barrels. Second, the sources should test the different aspects of the imaging and reconstruction process. A hardwaresoftware design which does a good job assaying a well chosen suite of sources should do a good job assaying almost any sources we might expect to encounter.

\section{Description of line, uniform, and can sources.}

We chose three simple sources for our design study. The first source is a line source. In relation to the instrument response, we would expect that assays made of a line source would be the least sensitive to resolution. For a line source, hardware designs with low resolution should work quite well. The line source also serves as a useful diagnostic on the test procedure since the expected response of the instrument to a line source is easily calculated by hand. The second type of source is a uniform concentration of radioactive material in a glass matrix as might be found in vitrified or otherwise solidified liquid waste. Despite the simplicity of the source, this is nonetheless a difficult assay problem because the attenuation of the emitted radiation is so severe. For example each inch of glass will absorb about $20 \%$ of the radiation with an energy of $414 \mathrm{keV}$, a useful energy for Plutonium. Radiation from the center of a 24 " diameter glass cylinder will be reduced by about a factor of 10 . The software reconstruction must do a good job of correcting the apparent radioactivity in the center of the cylinder to generate an accurate assay. The third source chosen is a 4 inch diameter radioactive plastic cylinder inside a thick walled aluminum can. While we might not expect to find exactly this item in a waste barrel, this source is representative of the type of source where spatial resolution is important in making an accurate assay. For example, at low resolution, the detector will not be able to discriminate the wall of the can, with its higher attenuation, from the radioactive source with its lower self-attenuation. The data will blend the regions of emission and high and low attenuation together and it will be impossible for any reconstruction algorithm to properly correct the measured activity for absorption. Thus we expect the canned source to be most sensitive to resolution. 


\section{Design Study}

In our preliminary design study we tested the three parameters, collimator width and length, and sampling interval against the three sources using an LLNL simulator to generate images of the absorbers [AZE90], the UCSF multi-ray simulator to generate emission data, and the UCSF MLEM reconstruction code to make the source images. The simulated emission data was produced from images of the absorbers and the sources which had grid size at least 4 times finer than the reconstruction grid. In all cases the reconstruction grid was the same size as the collimator width. The absorption maps used in the reconstruction were first degraded to the same resolution as the reconstruction grid since the A\&PCT scanner will acquire absorption data using the same detector with the same resolution as used to acquire the emission data.

Both the uniform and canned source assume $0.1 \mathrm{mCi} \mathrm{Pu}$ per gram of glass or plastic, or a factor of 1000 higher than the maximum threshold for low level waste. The line source is $2.3 \mathrm{Ci}$ or $0.1 \mathrm{mCi} / \mathrm{gm}$ assuming a barrel weight of $50 \mathrm{lbs}$. In each experiment, the barrels, assumed to be 24 "x 36 " are scanned for a fixed time. The barrel is viewed from a number of angles. There are about 1.5 times as many angular views as spatial positions of the detector. For example, a scan with a 1" square collimator and 1" spacing will require 24 positions to cover the diameter of the barrel. In this case there will be 36 views at 10 degree increments. If the barrel is scanned for 6 hour, there will be 0.7 seconds for each detector position or measurement. With a 2" square aperture there would be 8 seconds per position. Even though the study is done in only $2 \mathrm{D}$, in scaling the counts, we allow time for measurements in the third dimension. In order to produce an idea of the errors due to low signal-to-noise the reconstruction of the simulated data was run 7 times for each experiment with different realizations of Poisson noise added to the data, that is the sinogram, before each reconstruction.

Figures 2 through 4 show the results of the study. As we expect, the experiments with high spatial resolution and a low number of photon counts in the sinogram show a large spread in the assay values, but centered on the correct value. The experiments made with low resolution and high $S / N$ per measurement, show little statistical variation, but often a large error or bias. These errors relate to the poor resolution of the sources and absorbers. The best designs minimize both types of error. Figure 2 which shows experiments of 3 collimator widths for a fixed collimator length of $10^{\text {" }}$ indicates that of the 3 widths, 1", 2", and 4", the best results for our three sources are obtained with a $2 "$ aperture. Figure 3 shows that increasing the collimator length to $20^{\prime \prime}$, higher resolution, improves the mean accuracy, 
especially for the canned source, but also increases the statistical variation. Figure 4 shows that increasing the sampling interval of the 1 " aperture to 2 " improves the overall accuracy because the counting statistics improve. Further undersampling degrades the accuracy. Increasing the sampling of the $2 "$ aperture to 4 " results in some slight loss of accuracy in the mean, although with less statistical variation. Taken together, these figures suggest a collimator width of 2" to $3 "$, a collimator of $10^{\prime \prime}$ to $15^{\prime \prime}$ and a sampling interval of 2" to 3". More designs could be simulated to refine these numbers, but our A\&PCT system will need to be designed for 3D imaging. These preliminary results are sufficient to demonstrate how to design the $3 \mathrm{D}$ scanner.

These results for simulated waste with an activity of $0.1 \mathrm{mCi} / \mathrm{gm}$ also show that assaying nuclear waste at the maximum threshold for low level waste of $100 \mathrm{nCi} / \mathrm{gm}$ is a difficult problem. If we were to decrease the activity in the simulations by a factor of 1000 , the dominant errors would be statistical erriors from the low count rate. We can improve the counting statistics by various means. For example, using more than one detector will improve the count rate proportional to the number of detectors. Undersampling will allow for an improvement in speed by a factor of 2 to 4 . Combining multiple lines of $\mathrm{Pu}$ which are close together in energy, for example the lines at 379 and $414 \mathrm{keV}$, will improve the count rate by the number of lines combined. Scanning longer will always help. For example, our study is valid for an activity of $100 \mathrm{nCi} / g m$ assuming a scanner with 12 detectors with 2" square collimators, undersampling by 2 , measuring 2 Pu lines, and using a 10 hour scan time rather than 1 hour.

\section{Conclusions}

1) We show how to explore the trade-off between resolution and signal-tonoise using simulations of different hardware designs and sources.

2) For nuclear waste barrels, preliminary results indicate that collimator widths of 1 to 3 " with collimator aspect ratios of 5:1 to 10:1 respectively will perform well for assay purposes. Modest undersampling with a 1 " collimator may improve the accuracy by allowing more time for each individual measurement.

3) By following the same methodology with 3D imaging and reconstruction we should be able to determine appropriate design parameters for the 3D scanner. 


\section{References}

[AZE90] Computed Tomography Software and Standards, S.G.

Azevedo, H.E. Martz, M.F. Skeate, D.J. Schneberk, and G.P. Roberson, Lawrence Livermore National Laboratory, Livermore, Calif., UCRL-ID-105132, February, 1990.

ROB94 "Characterization of Waste Drums Using Nonintrusive Active and Passive Computedd Tomography," G. Patrick Roberson, Harry E. Martz, Daniel J. Decman, David C. Camp, Stephen G. Azevedo, and Eric R. Keto, Proceedings of the Nondestructive Assay and Nondestructive Examination Waste Characterization Conference, Pocatello, Idaho, February 14-16, 1994; UCRL-JC118317, Lawrence Livermore National Laboratory, Livermore, CA, August 1994.

MAR92 "Experimental A\&PCT Research and Development Efforts to Characterize Mixed Waste Forms," H. E. Martz, G. P Roberson, C. Robert-Coutant, and D. C. Camp, Proceedings of the Transuranic Waste Characterization Conference, Idaho State University, Pocatello, Idaho, August 10-12, 1992; UCRL-JC-110826, Lawrence Livermore National Laboratory, Livermore, CA, December 1992.

BRO94 J.K. Brown and B.H. Hasegawa, "Optimized Multi-ray Projection Integrators for SPECT," submitted for publication.

BRI86 J. F. Briesmeister, Editor, "MCNP- A General Monte Carlo Code for Neutron and Photon Transport, Version 3A", Los Alamos National Laboratory Report LA-7396-M, Rev. 2 (1986)

SHE82 L.A. Shepp and Y. Vardi, "Maximum Likelihood Reconstruction for Emission Tomography", IEEE Trans. Medical Imaging, MI-1, No 2, 113, 1982 

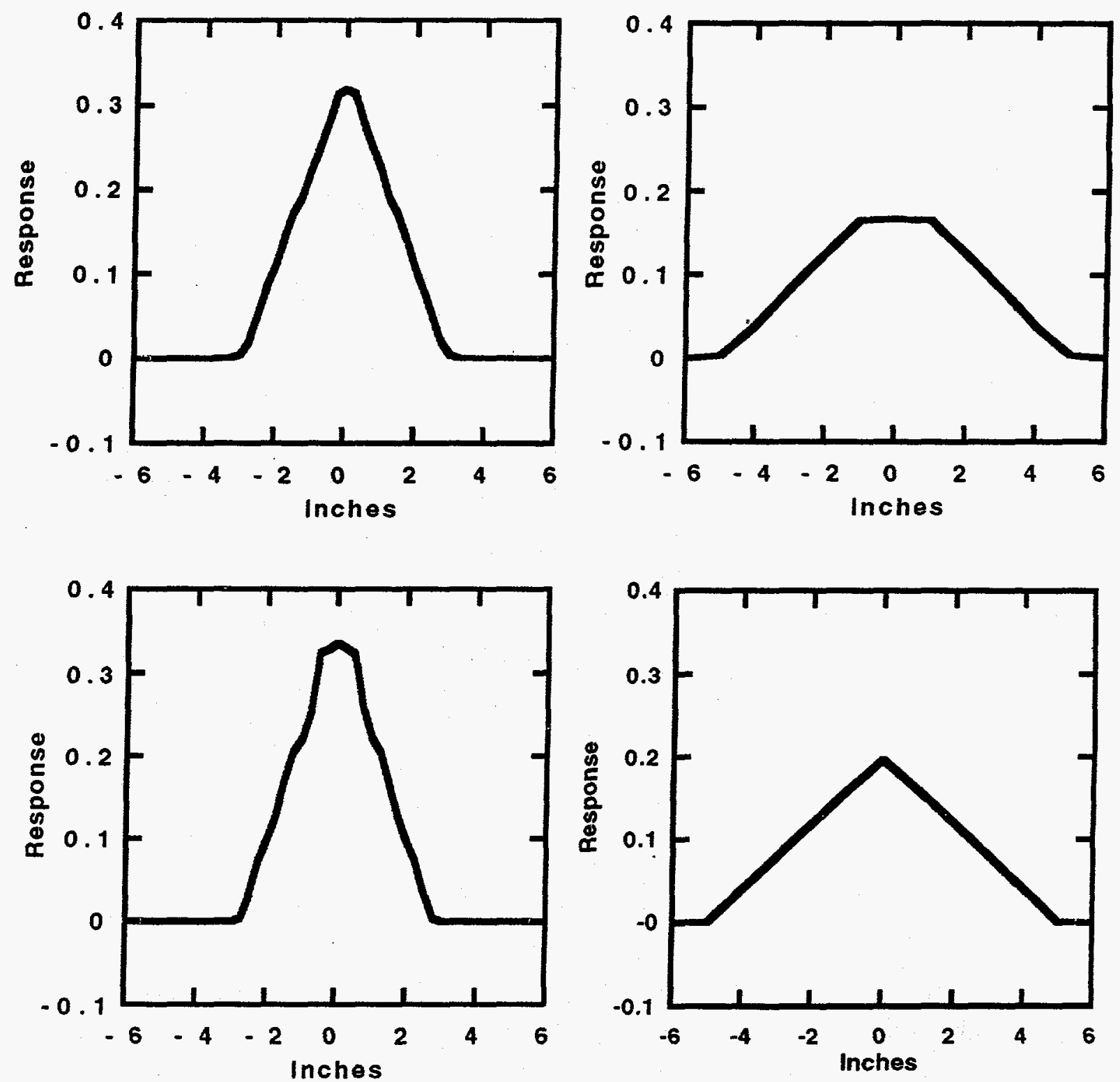

Figure 1: Comparison of instrumental response functions simulated by LLNL Monte Carlo modeling (bottom) and by UCSF multi-ray modeling (top). The simulated responses are for a high resolution $1 / 4^{\prime \prime}$ square collimator 5" long (left) and a low resolution 2" square 10" long (right). The comparison shows that the much simpler multi-ray code is capable of producing reasonable simulations. 


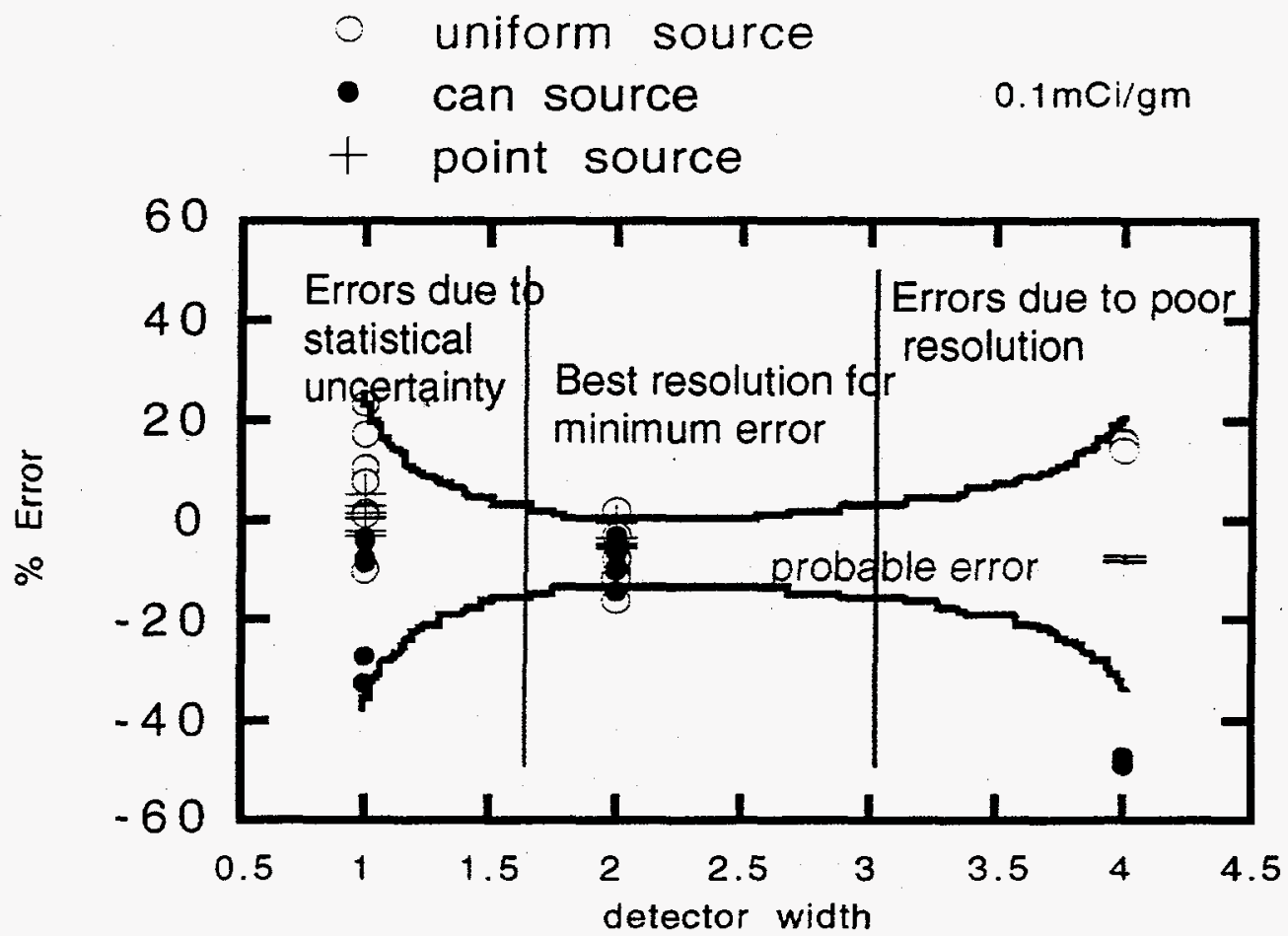

Figure 2: Expected assay accuracy for different collimator widths showing trade-off between spatial resolution and signal-to-noise. Small collimator widths which have high spatial resolution, on the right hand side of the figure, show large statistical errors due to the few number of counts collected. Large collimator widths with low spatial resolution show large errors in the assay due to inability to discriminate the individual emitting and absorbing structures. The data indicate a probable error envelope which can be roughly sketched in as shown. The most accurate assays are obtained with moderate resolution. A description of the source types, uniform, can, and line is in the text. 


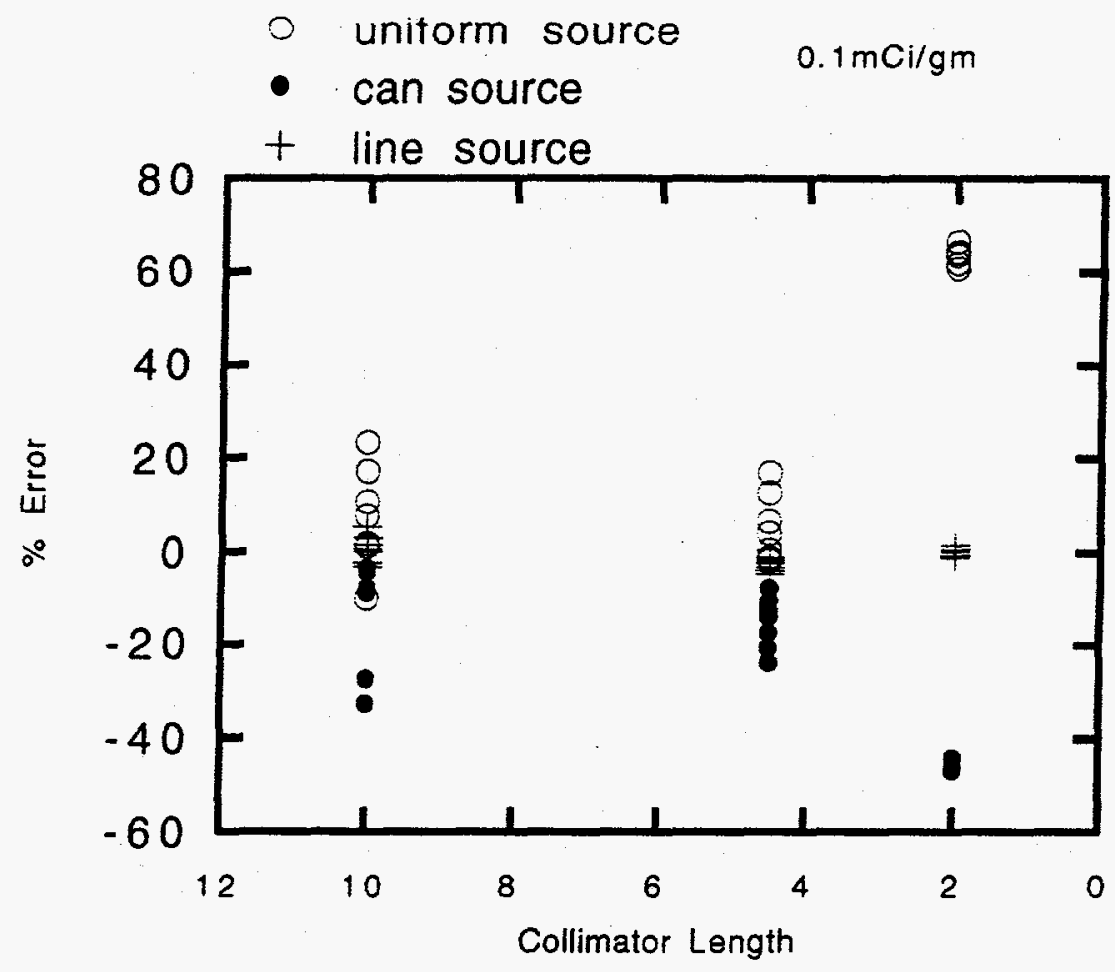

Figure 3: Comparison of accuracy of assays for different collimator lengths with a 1" square aperture. The collimator lengths are given on the horizontal axis. A description of the source types is in the text. The most accurate assays are obtained with moderate resolution. Sketching in the probable errors for each case will produce a graph similar to figure 2 . 


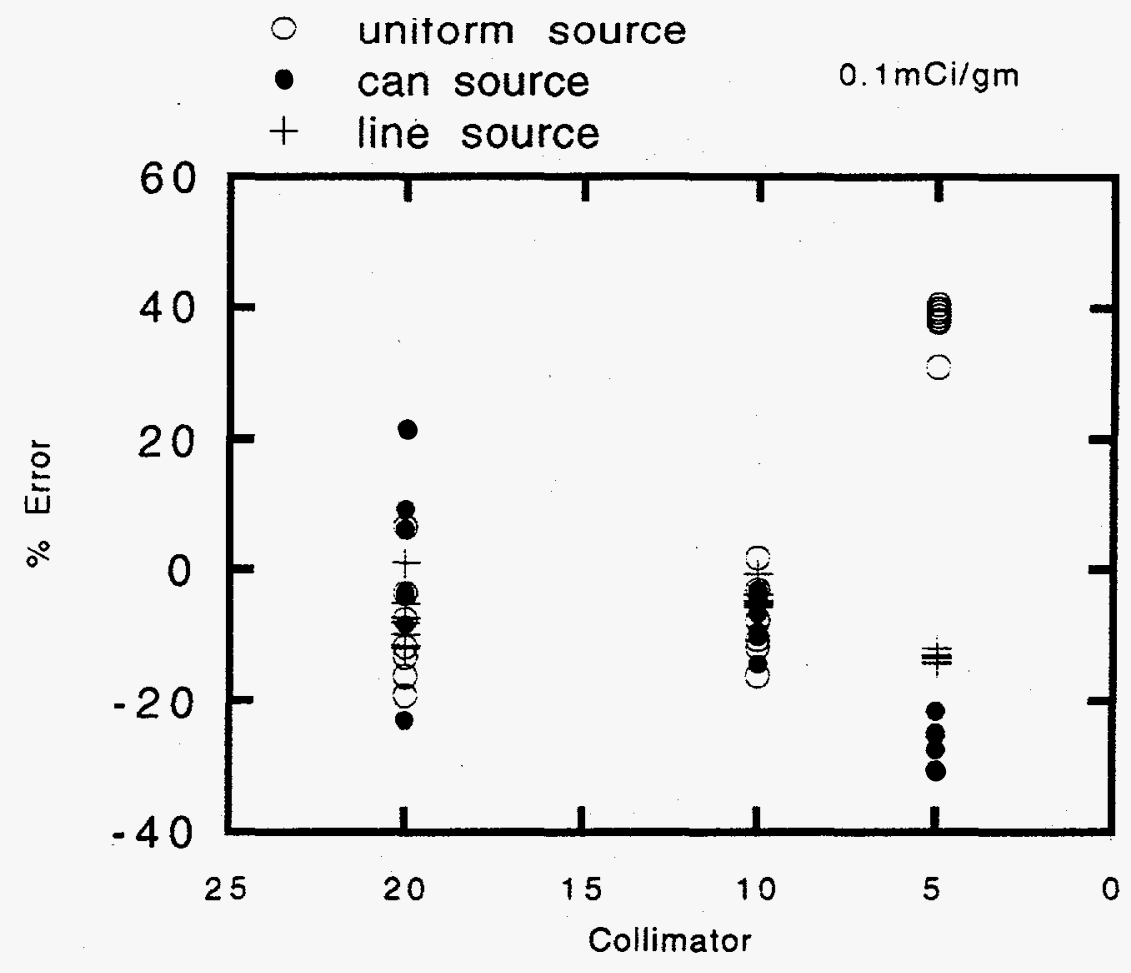

Figure 4: Comparison of accuracy of assays for different collimator lengths with a 2" square aperture. The collimator lengths are given on the horizontal axis. A description of the source types is in the text. The most accurate assays are obtained with moderate resolution. Sketching in the probable errors for each case will produce a graph similar to figure 2 . 


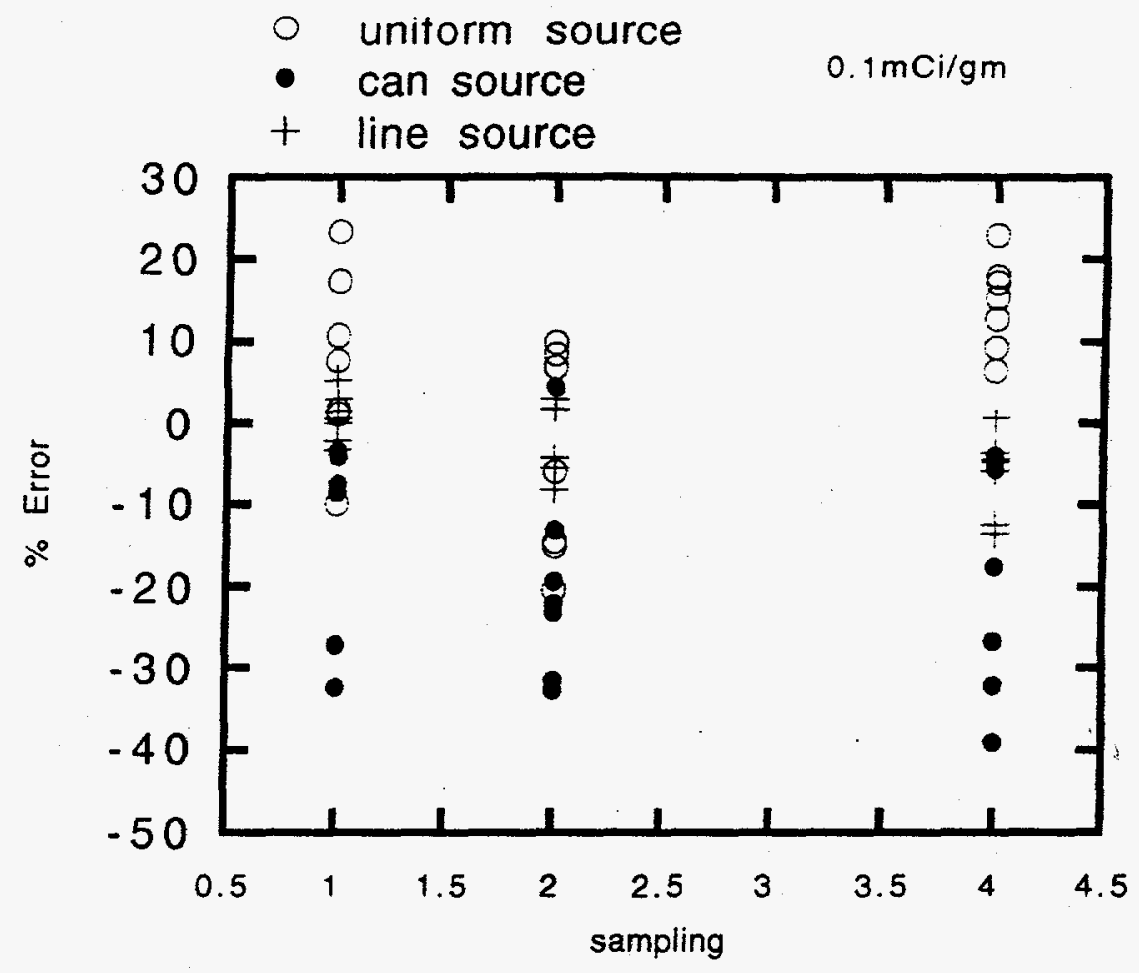

Figure 5: Comparison of accuracy of assays for different sampling intervals assuming a 1 " square aperture. The sampling intervals are given on the horizontal axis A description of the source types is in the text. This figure suggests that the assay accuracy of the high resolution collimator, 1", may be improved by undersampling by a factor of 2 . Sketching in the probable errors for each case will produce a graph similar to figure 2 . 


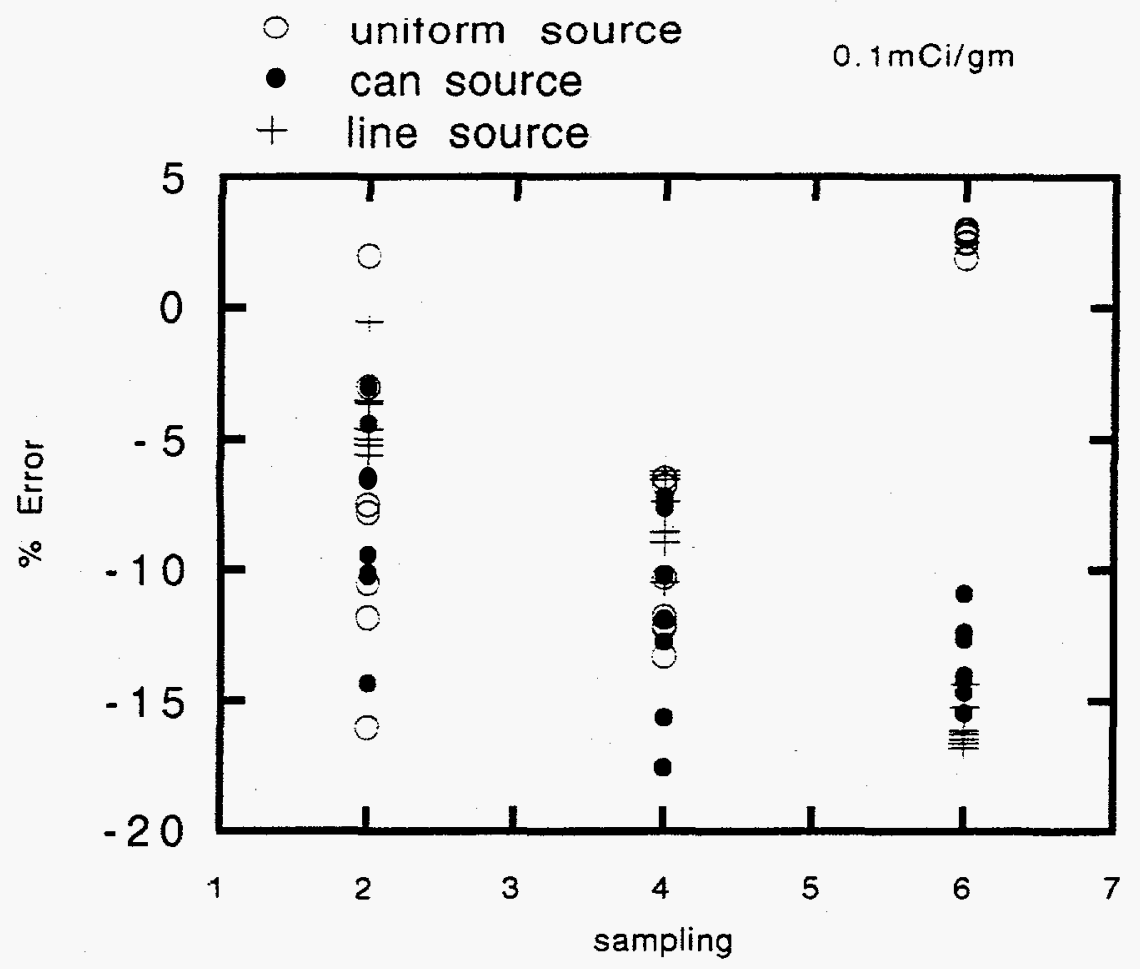

Figure 6: Comparison of accuracy of assays for different sampling intervals assuming a 2" square aperture. The sampling intervals are given on the horizontal axis A description of the source types is in the text. This figure suggests that the assay accuracy of the low resolution collimator, 2 " is not improved by undersampling. Sketching in the probable errors for each case will produce a graph similar to figure 2 . 